\title{
APPELL POLYNOMIAL EXPANSIONS AND BIORTHOGONAL EXPANSIONS IN BANACH SPACES
}

\author{
BY
}

\section{J. D. BUCKHOLTZ}

ABSTRACT. Let $\left\{p_{k}\right\}_{0}^{\infty}$ denote the sequence of Appell polynomials generated by an analytic function $\phi$ with the property that the power series for $\theta=1 / \phi$ has a larger radius of convergence than the power series for $\phi$. The expansion and uniqueness properties of $\left\{p_{k}\right\}$ are determined completely. In particular, it is shown that the only convergent $\left\{p_{k}\right\}$ expansions are basic series, and that there are no nontrivial representations of 0 . An underlying Banach space structure of these expansions is also studied.

1. Introduction. Let $a=\left\{a_{j}\right\}_{0}^{\infty}$ and $b=\left\{b_{j}\right\}_{0}^{\infty}$ be sequences of complex numbers which satisfy the formal power series identity

$$
\left(\sum_{j=0}^{\infty} a_{i} z^{j}\right)\left(\sum_{j=0}^{\infty} b_{j} z^{j}\right)=1 .
$$

The polynomials

$$
p_{k}(z)=\sum_{i=0}^{k} b_{k-j} \frac{z^{i}}{j !}, \quad k=0,1,2, \cdots
$$

are the Appell polynomials corresponding to the sequence $b$. They are biorthogonal to the sequence of linear functionals

$$
L_{k}(f)=\sum_{j=k}^{\infty} a_{j-k} f^{(j)}(0), \quad k=0,1,2, \cdots,
$$

i.e., $L_{k}\left(p_{j}\right)=0$ if $k \neq j$ and $L_{k}\left(p_{k}\right)=1$.

Every analytic function $f$ for which the functionals $L_{k}(f)$ are defined has the formal Appell expansion $f(z) \sim \sum_{k=0}^{\infty} L_{k}(f) p_{k}(z)$. Such expansions have been studied as part of a larger theory [1]; there is also an extensive literature dealing with specific polynomial sequences, e.g., the Bernoulli polynomials. In the case where each of $\phi(z)=\sum_{j=0}^{\infty} b_{j} z^{i}$ and $\theta(z)=\sum_{j=0}^{\infty} a_{j} z^{j}$ has a positive radius of convergence, Boas and Buck [1] have shown that the theory of entire functions of exponential type can be used to obtain deep results about such expansions.

Received by the editors July 27, 1972.

AMS (MOS) subject classifications (1970). Primary 30A16, 46B15, 46E 15; Secondary $12 \mathrm{E} 10$.

Key words and phrases. Appell polynomials, polynomial expansions, basic series, biorthogonal expansions, bases in Banach spaces. 
In the present paper, I employ infinite matrices of the form

$$
\left[\begin{array}{ccc}
b_{0}, & b_{1}, & b_{2}, \ldots \\
0, & b_{0}, & b_{1}, \ldots \\
0, & 0, & b_{0}, \ldots \\
\ldots \ldots & \ldots \ldots
\end{array}\right]
$$

to study these polynomial expansions. For a large class of such expansions, I am able to obtain complete results, and to present a portion of the theory in (presumably) its final form.

Let $\phi$ have radius of convergence $r(0<r<\infty)$ and no singularities other than poles on the circle $|z|=r$, and suppose that $\theta$ is analytic in the closed disk $|z| \leq r$. Throughout the remainder of the paper, we suppose that the polynomial sequence $\left\{p_{k}\right\}_{0}^{\infty}$ satisfies these conditions (no further hypotheses will be required).

We write $\phi(z)=\phi_{1}(z) / P(z)$, where $\phi_{1}$ is analytic and zero free in the closed disk $|z| \leq r$, and $P$ is a polynomial with all of its zeros on the circle $|z|=r$. Set

$$
P(z)=\prod_{q=1}^{\lambda}\left(1-\alpha_{q} z\right)^{m(q)},
$$

where $m(q)$ denotes the multiplicity of the zero $\alpha_{q}^{-1}$ of $P$, and let $m=$ $\max _{1 \leq q \leq \lambda} m(q)$. Essentially all of the expansion properties of the sequence $\left\{p_{k}\right\}_{0}^{\infty}$ can be obtained from the polynomial $P$; we set

$$
P_{t}(z)=\prod_{q=1}^{\lambda}\left(1-a_{q} z\right)^{\min \{t, m(q)\}} \quad \text { and } \quad Q_{t}(z)=\frac{P(z)}{P_{t}(z)}, \quad t=0,1, \cdots, m .
$$

(In case $m=1$, we adopt the convention that $Q_{m-2}=P$.) By a uniqueness set we mean a subset $E$ of the plane such that, if a solution $f$ of the differential equation $Q_{m-2}(D) f=0$ vanishes at every point of $E$, then $f \equiv 0$. Every infinite bounded set is a uniqueness set, as are "most" finite sets.

Convergence Theorem. If $b$ is a complex sequence, then the following are equivalent:

(i) each of the series

$$
\sum_{k=0}^{\infty}\left(\begin{array}{c}
k+m(q)-1 \\
m(q)-1
\end{array}\right) b_{k} \alpha_{q}^{k}, \quad 1 \leq q \leq \lambda
$$

converges;

(ii) for some fixed complex number $z_{0}$, each of the series

converges;

$$
\sum_{k=0}^{\infty} b_{k} p_{k}^{(n)}\left(z_{0}\right), \quad 0 \leq n<m \lambda
$$


(iii) the series

$$
S(z)=\sum_{k=0}^{\infty} b_{k} p_{k}(z)
$$

converges for all $z$ in some uniqueness set;

(iv) the series (1.1) converges for all $z$, the convergence being uniform on every compact set.

The proof of the Convergence Theorem does not require our hypothesis on the function $\theta$. This extends a result of Read [4] which states that uniform convergence of (1.1) on an open set implies uniform convergence on every compact set.

In order to characterize those functions $S$ which are the sum of a series of the form (1.1), more terminology is required. Write $P_{m-1}$ as $P_{m-1}(z)=$ $\Sigma_{k=0}^{\tau} C_{k} z^{k}$, and let $\mathcal{F}$ denote the space of entire functions $f$ such that

$$
\lim _{n \rightarrow \infty} \frac{f^{(n)}(0)}{r^{n}}=0 \quad \text { and } \quad \lim _{n \rightarrow \infty} \frac{n^{m-1}}{r^{n}} \sum_{k=0}^{\tau} C_{k} f^{(n+k)}(0)=0 .
$$

For $f \in \mathcal{F}$, set

$$
\|f\|=\sup _{0 \leq n<\infty}\left\{\frac{\left|f^{(n)}(0)\right|}{r^{n}}+\frac{\left(\begin{array}{c}
n+m-1 \\
m-1
\end{array}\right)}{r^{n}}\left|\sum_{k=0}^{\tau} C_{k} f^{(n+k)}(0)\right|\right\},
$$

where $\left(\begin{array}{c}n+m-1 \\ m-1\end{array}\right)$ denotes the binomial coefficient $(n+m-1) ! / n !(m-1) !$. The space $\mathcal{F}^{m-1}$ contains all entire functions of exponential type less than $r$, some functions of exponential type $r$, and no function of exponential type greater than $r$. Since $\left|f^{(n)}(0)\right| \leq\|f\| r^{n}, n=0,1,2, \cdots$, we have

$$
|f(z)| \leq \sum_{n=0}^{\infty}\left|f^{(n)}(0)\right| \frac{|z|^{n}}{n !} \leq\|f\| e^{r|z|}
$$

for every $f \in \mathcal{F}$ and every complex number $z$. Therefore convergence with respect to the norm $\|\cdot\|$ implies uniform convergence on compact sets.

Uniqueness Theorem. If $b$ is such that $S(z)=\sum_{k=0}^{\infty} b_{k} p_{k}(z)$ converges for all $z$, then $S \in \mathcal{F}$ and $b_{k}=L_{k}(S), k=0,1,2, \cdots$.

As a consequence of our results on convergence and uniqueness, the only series of the form (1.1) which need be considered as far as convergence is concerned are series of the form $\sum_{k=0}^{\infty} L_{k}(f)_{p_{k}}(z)$ for functions $f$ in the space $\mathcal{F}$. For such series, we settle the convergence question affirmatively. 
Expansion Theorem. If $f \in \mathcal{F}$, then $f(z)=\Sigma_{k=0}^{\infty} L_{k}(f) p_{k}(z)$ for all $z$, the convergence being uniform on every compact set. Furthermore,

for every $f \in \mathcal{F}$.

$$
\lim _{n \rightarrow \infty}\left\|f-\sum_{k=0}^{n} L_{k}(f) p_{k}\right\|=0
$$

The topology generated by the norm $\|\cdot\|$ is a great deal stronger than uniform convergence on compact sets. In some cases, $\mathcal{F}$ contains functions whose power series are not convergent with respect to this norm. There is no possibility, therefore, of deducing the Expansion Theorem by the usual technique of power series rearrangement.

The last question we study is that of the structure of the space $\mathcal{F}$. Two normed linear spaces $F$ and $G$ are said to be isomorphic if there is a 1-1 linear map $\pi$ of $F$ onto $G$ such that both $\pi$ and $\pi^{-1}$ are bounded. In the special case that $\phi$ has only simple poles on $|z|=r$, we have $m=1, P_{m-1}(z) \equiv 1$, and $\mathcal{F}$ is the space of all $f$ such that $\lim _{n \rightarrow \infty} f^{(n)}(0) / r^{n}=0$. In this case the norm is given by

$$
\|f\|=2 \sup _{0 \leq n<\infty}\left\{\frac{\left|f^{(n)}(0)\right|}{r^{n}}\right\} .
$$

An obvious isomorphism exists between this space and the Banach space $c_{0}$ of all complex sequences with limit 0 , normed with the supremum norm. The general case is far less obvious, but the result is the same.

Structure Theorem. The space $\mathfrak{F}$ is isomorphic to $c_{0}$.

It follows from the Structure Theorem that $\mathcal{F}$ is a Banach space, and from the Expansion and Uniqueness Theorems that the polynomials $\left\{p_{k}\right\}_{0}^{\infty}$ form a basis for this space. These theorems also imply that convergence in norm of $\sum_{k=0}^{\infty} b_{k} p_{k}$ is equivalent to conditions (i)-(iv) of the Convergence Theorem. The fact that $\left\{p_{k}\right\}_{0}^{\infty}$ is a basis for the Banach space $\mathcal{F}$ is the most fundamental expansion property of the polynomials $\left\{p_{k}\right\}_{0}^{\infty}$.

It is worth noting that there are no nontrivial representations of 0 of the form

$$
\sum_{k=0}^{\infty} b_{k} p_{k}(z) \text {. }
$$

If

$$
\sum_{k=0}^{\infty} b_{k} p_{k}(z)=0
$$

for all $z$ in a bounded infinite set, then the Convergence Theorem implies that 
(1.2) converges for all $z$ to an entire function $S$ that vanishes on a bounded infinite set. Therefore $S \equiv 0$. From the Uniqueness Theorem, we can conclude that $b_{k}=0, k=0,1,2, \cdots$. This result had been obtained previously by Read [4] under the additional assumptions that (1.3) holds uniformly on an open set and that $\theta$ is an entire function of finite exponential type.

The results obtained here for Appell polynomials can be extended to more general polynomials. The extension to Brenke polynomials $[1, \mathrm{p} .51]$ is almost trivial, and most of the results can, with slight modification, be extended to the generalized Appell expansions considered by W. T. Martin [3]. The Convergence Theorem is essentially due to Martin, who obtained a slightly weaker but considerably more general version [3].

2. Proof of the Convergence Theorem. We first obtain an asymptotic estimate for $p_{k}(z)$.

Lemma 2.1. If $\phi(z)=1 /(1-\alpha z)^{s+1}$, then

$$
p_{k}(z)=\alpha^{k} \sum_{t=0}^{s} \frac{(-1)^{t}}{t !}\left(\begin{array}{c}
k+s-t \\
s-t
\end{array}\right)(z / \alpha)^{t} \sum_{j=0}^{k-t} \frac{(z / \alpha)^{j}}{j !} .
$$

Also, the estimate

$$
\frac{p_{k}(z)}{\left(\begin{array}{c}
k+s \\
s
\end{array}\right) \alpha^{k} e^{z / a}}=1-\frac{s z}{a(k+s)}+O\left(k^{-2}\right), \quad k \rightarrow \infty,
$$

bolds uniformly on compact sets.

Proof. Since $1 /(1-\alpha z)^{s+1}=\sum_{k=0}^{\infty}\left(\begin{array}{c}(k+s) \\ s\end{array}\right) a^{k} z^{k}$, we have

$$
p_{k}(z)=\sum_{j=0}^{k}\left(\begin{array}{c}
k-j+s \\
s
\end{array}\right) a^{k-j} \frac{z^{j}}{j !}
$$

We use the binomial coefficient identity $\left(\begin{array}{c}k-j+s \\ s\end{array}\right)=\sum_{t=0}^{s}(-1)^{t}\left(\begin{array}{c}j \\ t\end{array}\right)\left(\begin{array}{c}k+s-t \\ s-t\end{array}\right)$ to obtain

$$
p_{k}(z)=\sum_{t=0}^{s}(-1)^{t}\left(\begin{array}{c}
k+s-t \\
s-t
\end{array}\right) \sum_{j=0}^{k} \alpha^{k-j}\left(\begin{array}{l}
j \\
t
\end{array}\right) \frac{z^{j}}{j !}=\alpha^{k} \sum_{t=0}^{s}(-1)^{\prime}\left(\begin{array}{c}
k+s-t \\
s-t
\end{array}\right) \sum_{j=t}^{k} \frac{(z / \alpha)^{j}}{t !(j-t) !} .
$$

Replacing $j$ by $j+t$ in the second sum produces the desired result. The asymptotic estimate follows easily from this.

For $1 \leq q \leq \lambda$, let

$$
\sum_{t=1}^{m(q)} \frac{c_{t}(q)}{\left(1-\alpha_{q} z\right)^{m(q)+1-t}}
$$


denote the sum of the negative powers in the Laurent expansion of $\phi$ at $\alpha_{q}^{-1}$. Note that $c_{1}(q) \neq 0,1 \leq q \leq \lambda$, and that the function $\phi_{2}$ defined by

$$
\phi(z)=\sum_{q=1}^{\lambda} \sum_{t=1}^{m(q)} \frac{c_{t}(q)}{\left(1-\alpha_{q} z\right)^{m(q)+1-t}}+\phi_{2}(z)
$$

is analytic in the closed disk $|z| \leq r$. The contribution to $p_{k}(z)$ of $\phi_{2}$ is given by

$$
\sum_{j=0}^{k} \frac{\phi_{2}^{(k-j)}(0)}{(k-j) !} \frac{z^{j}}{j !}
$$

we require an upper bound on the modulus of this quantity.

Lemma 2.2. There exist constants $K>0$ and $r_{1}>r$ such that

$$
\left|\sum_{j=0}^{k} \frac{\phi_{2}^{(k-j)}(0)}{(k-j) !} \frac{z^{j}}{j !}\right| \leq \frac{K e^{r_{1}|z|}}{r_{1}^{k}}
$$

bolds for all $z$ and every nonnegative integer $k$.

Proof. Choose $r_{1}>r$ such that $\phi_{2}$ is analytic in the closed disk $|z| \leq r_{1}$. Therefore

$$
\lim _{j \rightarrow \infty} \frac{\phi_{2}^{(j)}(0)}{j !} r_{1}^{j}=0
$$

set $K=\max _{0 \leq j<\infty}\left|\phi_{2}^{(j)}(0)\right| r_{1}^{j} / j !$. Then $\left|\phi_{2}^{(k-j)}(0)\right| /(k-j) ! \leq K / r_{1}^{k-j}$; and the result follows from the triangle inequality.

It is now an easy matter to verify the estimate contained in the following lemma, whose proof we omit.

Lemma 2.3. The asymptotic estimates

$$
\begin{aligned}
p_{k}(z)= & \left(\begin{array}{c}
k+m-1 \\
m-1
\end{array}\right) \sum_{m(q)=m} e^{z / a} q_{a}^{k}\left\{c_{1}(q)+\frac{(m-1) c_{2}(q)}{k+m-1}\right\} \\
& -\left(\begin{array}{c}
k+m-2 \\
m-2
\end{array}\right) \sum_{m(q)=m} z e^{z / a} a_{a}^{k} \frac{c_{1}(q)}{\alpha_{q}} \\
& +\left(\begin{array}{c}
k+m-2 \\
m-2
\end{array}\right) \sum_{m(q)=m-1} e^{z / a_{q}} \alpha_{q}^{k} c_{1}(q)+O\left(\frac{k^{m-3}}{r^{k}}\right) \\
= & \left(\begin{array}{c}
k+m-1 \\
m-1
\end{array}\right) \sum_{m(q)=m} e^{z / \alpha_{q}} \alpha_{q}^{k} c_{1}(q)+O\left(\frac{k^{m-2}}{r^{k}}\right)
\end{aligned}
$$

bold uniformly on every compact set. 
It is convenient to arrange the proof of the Convergence Theorem in the form

$$
\begin{gathered}
(\mathrm{i}) \Rightarrow \text { (iv) } \Rightarrow \text { (ii) } \Rightarrow \text { (i) } \\
\Downarrow \\
(\text { iii }) \Rightarrow(\text { i }) .
\end{gathered}
$$

The implications (iv) $\Rightarrow$ (ii) and (iv) $\Rightarrow$ (iii) are obvious. We now show that (i) $\Rightarrow$ (iv). For each $k$, let $g_{k}(z)$ denote the quantity which appears in Lemma 2.3 under the name $O\left(k^{m-3 / r^{k}}\right)$. Since $\sum_{k=0}^{\infty}\left(\begin{array}{c}k+m-1 \\ m-1\end{array}\right) b_{k} a_{q}^{k}$ converges for some value of $q$, we have $\lim _{k \rightarrow \infty}\left(\begin{array}{c}k+m-1 \\ m-1\end{array}\right) b_{k} a_{q}^{k}=0$. Therefore

$$
b_{k}=o\left(r^{k} / k^{m-1}\right) \quad \text { and } \quad b_{k} g_{k}(z)=o\left(k^{-2)}, \quad k \rightarrow \infty,\right.
$$

uniformly on compact sets. Consequently, $\sum_{k=0}^{\infty} b_{k} g_{k}(z)$ is uniformly convergent on compact sets, and it only remains to prove that the same is true of

$$
\sum_{k=0}^{\infty} b_{k}\left\{p_{k}(z)-g_{k}(z)\right\}
$$

Since

$$
\left(\begin{array}{c}
k+m-2 \\
m-2
\end{array}\right)=\frac{m-1}{k+m-1}\left(\begin{array}{c}
k+m-1 \\
m-1
\end{array}\right)
$$

it is not hard to show that the convergence of $\sum_{k=0}^{\infty} \underset{m-1}{(k+m-1)} b_{k} \alpha_{q}^{k}$ implies the convergence of

$$
\sum_{k=0}^{\infty}\left(\begin{array}{c}
k+m-2 \\
m-2
\end{array}\right) b_{k} \alpha_{q}^{k}
$$

we shall give a proof for this later. Taking this for granted temporarily, (i) obviously implies the uniform convergence on compact sets of the series

$$
\begin{gathered}
c_{1}(q) e^{z / a_{q}} \sum_{k=0}^{\infty}\left(\begin{array}{c}
k+m-1 \\
m-1
\end{array}\right) b_{k} \alpha_{q}^{k} \quad(m(q)=m), \\
c_{2}(q) e^{z / a} q \sum_{k=0}^{\infty}\left(\begin{array}{c}
k+m-2 \\
m-2
\end{array}\right) b_{k} \alpha_{q}^{k} \quad(m(q)=m), \\
-\frac{c_{1}(q)}{\alpha_{q}} z e^{z / a_{q}} \sum_{k=0}^{\infty}\left(\begin{array}{c}
k+m-2 \\
m-2
\end{array}\right) b_{k} \alpha_{q}^{k} \quad(m(q)=m), \quad \text { and } \\
c_{1}(q) e^{z / a} \sum_{k=0}^{\infty}\left(\begin{array}{c}
k+m-2 \\
m-2
\end{array}\right) b_{k} a_{q}^{k} \quad(m(q)=m-1) .
\end{gathered}
$$


Since (2.1) is a finite sum of such series, it, too, is uniformly convergent on compact sets; this proves that (i) implies (iv).

Before proving that (iii) implies (i), we need a result on linearly independent sets of functions.

Lemma 2.4. Suppose $\left\{f_{q}\right\}_{1}^{t}$ is a linearly independent set of functions with common domain $\Omega$. If $E$ is a subset of $\Omega$ such that the functions $\left\{f_{q}\right\}_{1}^{t}$ restricted to $E$ are linearly independent, then there are points $\left\{z_{j}\right\}_{1}^{t}$ in $E$ such that the determinant $\operatorname{det}\left\{f_{q}\left(z_{j}\right)\right\}, 1 \leq q, j \leq t$, does not vanish.

The proof is easy and is omitted.

Lemma 2.5. If $S(z)=\sum_{k=0}^{\infty} b_{k} p_{k}(z)$ converges at every point of some uniqueness set $E$, then $b_{k}=o\left(r^{k} / k^{m-1}\right), k \rightarrow \infty$.

Proof. Let the zeros of $P$ be ordered so that $m(q)=m$ if and only if $1 \leq$ $q \leq t(t \leq \lambda)$. The functions $c_{1}(q) e^{z / \alpha_{q}}, 1 \leq q \leq t$, are linearly independent solutions of the differential equation $Q_{m-2}(D) f=0$ (recall that $c_{1}(q) \neq 0$ ). Therefore their restrictions to $E$ are linearly independent, and we can find points $\left\{z_{j}\right\}_{1}^{t}$ in $E$ such that $\delta_{t}=\operatorname{det}\left\{c_{1}(q) e^{z_{j} / a_{q}}\right\} \neq 0$.

Since each of $\Sigma_{k=0}^{\infty} b_{k} p_{k}\left(z_{j}\right), 1 \leq j \leq t$, converges, their terms tend to 0 , and we have

$$
\lim _{k \rightarrow \infty}\left|b_{k}\right| \sum_{j=1}^{t}\left|p_{k}\left(z_{j}\right)\right|=0
$$

Set

$$
p_{k}(z)=\left(\begin{array}{c}
k+m-1 \\
m-1
\end{array}\right) \sum_{q=1}^{t} c_{1}(q) e^{z / a} q_{\alpha} a_{q}^{k}+u_{k}(z)
$$

then

$$
u_{k}(z)=o\left(k^{m-1 / r^{k}}\right)
$$

by Lemma 2.3. Let

$$
v_{k}(z)=\left\{p_{k}(z)-u_{\dot{p}}(z)\right\} \frac{r^{k}}{\left(\begin{array}{c}
k+m-1 \\
m-1
\end{array}\right)} .
$$

Since $\delta_{t} \neq 0$, the system of equations

$$
v_{k}\left(z_{j}\right)=\sum_{q=1}^{t} c_{1}(q) e^{z_{j} / \alpha_{q}}\left(\alpha_{q} r\right)^{k}, \quad 1 \leq j \leq t
$$


can be "solved" for the quantities $\left(a_{q} r\right)^{k}$ in terms of $v_{k}\left(z_{j}\right), 1 \leq j \leq t$. In particular, there exist constants $M_{j}(1 \leq j \leq t)$ such that

$$
\left(a_{1} r\right)^{k}=\sum_{j=1}^{t} M_{j} v_{k}\left(z_{j}\right)
$$

Set $M=\max \left|M_{j}\right|, 1 \leq j \leq t$. If in (2.5) we take absolute values and apply the triangle inequality, we obtain $1 \leq M \sum_{j=1}^{t}\left|v_{k}\left(z_{j}\right)\right|$. From the definition of $v_{k}$ and (2.4), we see that

$$
\sum_{j=1}^{t}\left|p_{k}\left(z_{j}\right)\right|>\frac{\left(\begin{array}{c}
k+m-1) \\
m-1
\end{array}\right.}{2 M r^{k}}
$$

holds for all $k$ sufficiently large. Therefore

$$
\underset{k \rightarrow \infty}{\lim \sup } \frac{k^{m-1}}{r^{k}}\left\{\sum_{j=1}^{t}\left|p_{k}\left(z_{j}\right)\right|\right\}^{-1}<\infty .
$$

The desired result now follows from (2.3) and (2.6).

The following lemma is equivalent to the fact that (iii) implies (i).

Lemma 2.6. If

$$
S(z)=\sum_{k=0}^{\infty} b_{k} p_{k}(z)
$$

converges for all $z$ in some uniqueness set $E$, then each of the series

$$
\sum_{k=0}^{\infty}\left(\begin{array}{c}
k+m(q)-1 \\
m(q)-1
\end{array}\right) b_{k} \alpha_{q}^{k}, \quad 1 \leq q \leq \lambda,
$$

converges.

Proof. In view of Lemma 2.5, (2.8) clearly converges if $m(q)<m-1$. Also, from Lemmas 2.3 and $2.5,(2.7)$ is convergent if and only if $\sum_{k=0}^{\infty} b_{k}\left\{p_{k}(z)-g_{k}(z)\right\}$ is convergent, where $g_{k}$ is defined as before.

Let $\left\{f_{t}(z)\right\}_{1}^{s}$ denote the solutions of the differential equation $Q_{m-2}(D) f=0$ which are of the forms $e^{z / \alpha_{q}}$ and $z e^{z / a_{q}}$. From the decomposition of $p_{k}$ in Lemma 2.3, we have

$$
T_{n}(z)=\sum_{k=0}^{n} b_{k}\left\{p_{k}(z)-g_{k}(z)\right\}=\sum_{t=1}^{s} f_{t}(z) U_{t}(n),
$$

where 


$$
\begin{gathered}
U_{t}(n)=\sum_{k=0}^{n}\left(\begin{array}{c}
k+m-1 \\
m-1
\end{array}\right) b_{k} \alpha_{q}^{k}\left\{c_{1}(q)+\frac{(m-1) c_{2}(q)}{k+m-1}\right\} \\
\text { if } f_{t}(z)=e^{z / a} q \text { and } m(q)=m, \\
U_{t}(n)=-\frac{c_{1}(q)}{\alpha_{q}} \sum_{k=0}^{n}\left(\begin{array}{c}
k+m-2 \\
m-2
\end{array}\right) b_{k} \alpha_{q}^{k} \text { if } f_{t}(z)=z e^{z / \alpha_{q}} \text { and } m(q)=m, \quad \text { and } \\
U_{t}(n)=c_{1}(q) \sum_{k=0}^{n}\left(\begin{array}{c}
k+m-2 \\
m-2
\end{array}\right) b_{k} \alpha_{q}^{k} \text { if } f_{t}(z)=e^{z / \alpha_{q}} \text { and } m(q)=m-1 .
\end{gathered}
$$

Choose points $\left\{z_{j}\right\}_{1}^{s}$ in $E$ such that the determinant $\delta_{s}=\operatorname{det}\left\{f_{t}\left(z_{j}\right)\right\}$ does not vanish. We can then solve the system of equations

$$
T_{n}\left(z_{j}\right)=\sum_{t=1}^{s} f_{t}\left(z_{j}\right) U_{t}(n), \quad 1 \leq j \leq s,
$$

and express each $U_{t}(n)$ as a linear combination of the quantities $T_{n}\left(z_{j}\right), 1 \leq$ $j \leq s$. Since each of the latter possesses a limit as $n \rightarrow \infty$, the same is true of the quantities $U_{t}(n), 1 \leq t \leq s$. Therefore,

$$
\sum_{k=0}^{\infty}\left(\begin{array}{c}
k+m-1 \\
m-1
\end{array}\right) b_{k} \alpha_{q}^{k}\left\{c_{1}(q)+\frac{(m-1) c_{2}(q)}{k+m-1}\right\}
$$

converges if $m(q)=m$, and $\sum_{k=0}^{\infty} \underset{m-2}{(k+m-2)} b_{k} \alpha_{q}^{k}$ converges if $m(q)=m-1$. We shall show later that the convergence of (2.9) is equivalent to the convergence of

$$
\sum_{k=0}^{\infty}\left(\begin{array}{c}
k+m-1 \\
m-1
\end{array}\right) b_{k} \alpha_{q}^{k},
$$

and this will complete the proof of Lemma 2.6.

A complex sequence $x$ is said to be of bounded variation (absolutely convergent) if the quantity $V(x)=\left|x_{0}\right|+\sum_{k=1}^{\infty}\left|x_{k}-x_{k-1}\right|$ is finite; $V(x)$ is called the total variation of $x$. Sequences of bounded variation are necessarily convergent; their properties. we shall use most often are contained in the following two lemmas.

Lemma 2.7. Suppose that $x$ and $y$ are complex sequences such that $x$ is of bounded variation and $\Sigma_{0}^{\infty} y_{k}$ converges. Then $\Sigma_{0}^{\infty} x_{k} y_{k}$ converges,

$$
\sum_{k=0}^{\infty} x_{k} y_{k}=x_{0} \sum_{j=0}^{\infty} y_{j}+\sum_{k=1}^{\infty}\left(x_{k}-x_{k-1}\right) \sum_{j=k}^{\infty} y_{j},
$$

and 


$$
\left|\sum_{k=0}^{\infty} x_{k} y_{k}\right| \leq V(x) \sup _{0 \leq k<\infty}\left|\sum_{j=k}^{\infty} y_{j}\right| .
$$

Lemma 2.7 is a well known extension of Abel's identity; its proof is easy and is omitted.

Lemma 2.8. Suppose that $x$ is of bounded variation and $\lim x_{k} \neq 0$. If $y$ is a complex sequence, then $\sum_{k=0}^{\infty} y_{k}$ converges if and only if $\sum_{k=0}^{\infty} x_{k} y_{k}$ converges.

Proof. Lemma 2.7 guarantees that convergence of the first series implies the convergence of the second. Choose $K$ so that $x_{k} \neq 0$ for $k \geq K$. The sequence $\left\{1 / x_{k}\right\}_{k=K}^{\infty}$ is of bounded variation; therefore convergence of $\sum_{k=K}^{\infty} x_{k} y_{k}$ implies the convergence of $\sum_{k=K}^{\infty} y_{k}$, and the proof is complete.

The convergence of (2.2), which we assumed previously, follows from the fact that $\{(m-1) /(k+m-1)\}_{k=0}^{\infty}$ is of bounded variation. To deduce the convergence of (2.10) from that of (2.9), note that

$$
\left\{c_{1}(q)+(m-1) c_{2}(q) /(k+m-1)\right\}_{k=0}^{\infty}
$$

is of bounded variation and has limit $c_{1}(q) \neq 0$.

We now prove that (ii) implies (i), which will complete the proof of the Convergence Theorem.

Lemma 2.9. Suppose $\lambda=1$. If $z_{0}$ is a complex number and $b$ is a complex sequence such that

$$
\sum_{k=0}^{\infty} b_{k} p_{k}\left(z_{0}\right)
$$

converges, then

$$
\sum_{k=0}^{\infty}\left(\begin{array}{c}
k+m(1)-1 \\
m(1)-1
\end{array}\right) b_{k} \alpha_{1}^{k}
$$

converges.

Proof. If $\lambda=1$, then $m(1)=m$, and

$$
\begin{aligned}
p_{k}\left(z_{0}\right)= & \left(\begin{array}{c}
k+m-1 \\
m-1
\end{array}\right) e^{z_{0} / a_{1} \alpha_{1}^{k}}\left\{c_{1}(1)+\frac{(m-1)\left(c_{2}(1)-z_{0} c_{1}(1) / \alpha_{1}\right)}{k+m-1}\right\} \\
& +o\left(\frac{k^{m-3}}{r^{k}}\right), \quad k \rightarrow \infty,
\end{aligned}
$$

from Lemma 2.3. Let 


$$
x_{k}=\frac{p_{k}\left(z_{0}\right)}{\left(\begin{array}{c}
k+m(1)-1) \alpha_{1}^{k} \\
m(1)-1
\end{array}\right.}=e^{z_{0} / a_{1}}\left\{c_{1}(1)+\frac{(m-1)\left(c_{2}(1)-z_{0} c_{1}(1) / \alpha_{1}\right)}{k+m-1}\right\}+O\left(k^{-2)} .\right.
$$

One sees, therefore, that $x$ is a sequence of bounded variation with limit $c_{1}(1) e^{z_{0} / \alpha_{1}} \neq 0$. Therefore the convergence of (2.11) is equivalent to that of (2.12) and the proof is complete.

Lemma 2.9 guarantees that (ii) implies (i) if the polynomial $P$ has only one zero (of arbitrary multiplicity). The proof in general amounts to reducing it to this case.

It is easy to verify that $p_{k}^{(n)}(z)=p_{k-n}(z)$ for every nonnegative integer $n$ (we adopt the convention that $p_{k-n}(z) \equiv 0$ if $k<n$ ). The other property of Appell polynomials which we need is contained in the following lemma.

Lemma 2.10. Suppose that $\left\{A_{n}\right\}_{0}^{s}$ is a finite sequence of complex numbers, and set $\rho_{k}(z)=\sum_{n=0}^{s} A_{n} p_{k-n}(z), k=0,1,2, \ldots$. Then $\left\{\rho_{k}\right\}_{0}^{\infty}$ is the sequence of Appell polynomials corresponding to the sequence $B_{k}=\sum_{n=0}^{\min }\{s, k\} A_{n} b_{k-n}, k=0$, $1,2, \cdots$. The function corresponding to $\phi$ is given by $\Phi(z)=\phi(z) \sum_{n=0}^{s} A_{n} z^{n}$.

The proof of Lemma 2.10 is a straightforward calculation and is omitted.

We now prove that (ii) implies (i). Suppose that $z_{0}$ is a complex number, $b$ is a complex sequence, and each of the series $\sum_{k=0}^{\infty} b_{k} p_{k}^{(n)}\left(z_{0}\right), 0 \leq n<m \lambda$, converges. Let $q$ be fixed $(1 \leq q \leq \lambda)$, and set $P(z) /\left(1-\alpha_{q} z\right)^{m(q)}=\sum_{n=0}^{s} A_{n} z^{n}$. Since the degree of $P$ is at most $m \lambda$, we have $s<m \lambda$. Let

$$
\rho_{k}(z)=\sum_{n=0}^{s} A_{n} p_{k-n}(z)=\sum_{n=0}^{s} A_{n} p_{k}^{(n)}(z), \quad k=0,1, \cdots
$$

Now

$$
\sum_{n=0}^{s} A_{n} \sum_{k=0}^{\infty} b_{k} p_{k}^{(n)}\left(z_{0}\right)=\sum_{k=0}^{\infty} b_{k} \sum_{n=0}^{s} A_{n} p_{k}^{(n)}\left(z_{0}\right)=\sum_{k=0}^{\infty} b_{k} \rho_{k}\left(z_{0}\right)
$$

so that the last series is convergent. On the other hand, $\left\{\rho_{k}\right\}_{0}^{\infty}$ is an Appell sequence, and the function that corresponds to $\phi$ for this sequence is

$$
\Phi(z)=\phi(z) \sum_{n=0}^{s} A_{n} z^{n}=\frac{\phi_{1}(z)}{P(z)} \frac{P(z)}{\left(1-\alpha_{q} z\right)^{m(q)}}=\frac{\phi_{1}(z)}{\left(1-\alpha_{q} z\right)^{m(q)}} .
$$

Therefore

$$
\sum_{k=0}^{\infty}\left(\begin{array}{c}
k+m(q)-1 \\
m(q)-1
\end{array}\right) b_{k} \alpha_{q}^{k}
$$

converges by Lemma 2.9 , and the proof is complete. 
3. The Mapping Theorem. Let $B$ denote the infinite matrix with entries $B_{j k}=p_{k}^{(j)}(0), 0 \leq j, k<\infty$. The domain of $B$ is the collection of all sequences $b$ such that each of

$$
\sum_{k=j}^{\infty} B_{j k} b_{k}=\sum_{k=j}^{\infty} b_{k} p_{k}^{(j)}(0), \quad j=0,1, \cdots,
$$

converges. The range of $B$ is the set of all sequences $y$ such that $y_{j}=$ $\sum_{k=j}^{\infty} B_{j k} b_{k}, j=0,1, \ldots$, for some $b$ in the domain of $B$. For our purposes, it is unnecessary to make a distinction between $B$ and the sequence to sequence operator it defines. Suppose that

$$
f(z)=\sum_{k=0}^{\infty} b_{k} p_{k}(z)
$$

holds uniformly on some neighborhood of 0 , and let $y=\left\{f^{(j)}(0)\right\}_{0}^{\infty}$. If we differentiate (3.1) $j$ times and set $z=0$, we obtain

$$
y_{j}=f^{(j)}(0)=\sum_{k=j}^{\infty} b_{k} p_{k}^{(j)}(0)=\sum_{k=j}^{\infty} B_{j k} b_{k}
$$

Therefore $b$ belongs to the domain of $B$ and $y$ belongs to the range of $B$. If

$$
f(z)=\sum_{k=0}^{\infty} L_{k}(f) p_{k}(z)
$$

holds uniformly on a neighborhood of 0 , a similar argument yields

$$
y=B(A y),
$$

where $A$ denotes the upper triangular matrix inverse of $B$. Note that $L_{k}(f)=$ $(A y)_{k}, k=0,1, \cdots$.

The preceding remarks apply not only to Appell polynomials, but to arbitrary polynomial sequences $\left\{p_{k}\right\}_{0}^{\infty}$ such that $p_{k}$ is of degree $k$. For reasonably wellbehaved polynomial sequences, one would expect that the necessary conditions (3.2) and (3.4) for (3.1) and (3.3) to hold might also be sufficient, and, frequently, this is the case [2]. We shall prove that this is true for all of the Appell sequences under consideration, and give an explicit determination of the range of $B$.

Let $Y$ denote the space of all sequences $y$ such that $y=\left\{f^{(j)}(0)\right\}_{0}^{\infty}$ for an $f \in \mathcal{F}$. Specifically, $Y$ is the space of all sequences $y$ such that

$$
\lim _{n \rightarrow \infty} \frac{y_{n}}{r^{n}}=0 \text { and } \lim _{n \rightarrow \infty} \frac{n^{m-1}}{r^{n}} \sum_{k=0}^{\tau} C_{k} y_{n+k}=0 .
$$


We endow $Y$ with the norm

$$
\|y\|_{Y}=\sup _{0 \leq n<\infty}\left\{r^{-n}\left|y_{n}\right|+\left(\begin{array}{c}
n+m-1 \\
m-1
\end{array}\right) r^{-n}\left|\sum_{k=0}^{\tau} C_{k} y_{n+k}\right|\right\} .
$$

With this norm, the identification of entire functions $f$ with sequences $\left\{f^{(j)}(0)\right\}_{0}^{\infty}$ induces a linear isometry between the spaces $\mathcal{F}$ and $Y$. Under this identification, the function $z^{k} / k$ ! is identified with the "unit coordinate vector" $e_{k}=\left\{\delta_{k j}\right\}_{j=0}^{\infty}$ $\left(\delta_{k j}\right.$ denotes the Kronecker delta), and the polynomial $p_{k}$ corresponds to the sequence $B e_{k}$. The expansion $f \sim \sum_{k=0}^{\infty} L_{k}(f) p_{k}$ corresponds to $y \sim \sum_{k=0}^{\infty}(A y)_{k} B e_{k}$.

It is considerably easier to investigate the expansion in the sequence space setting, and then use the isometry to translate results back into the analytic function setting.

Let $H$ denote the space of sequences $b$ such that (i) of the Convergence Theorem holds. For $b \in H$ set

$$
\|b\|_{H}=\max _{1 \leq q<\lambda} \sup _{0 \leq n<\infty}\left|\sum_{k=n}^{\infty}\left(\begin{array}{c}
k+m(q)-1 \\
m(q)-1
\end{array}\right) b_{k} \alpha_{q}^{k}\right| .
$$

The spaces $H$ and $Y$ are needed to describe the behavior of the matrix $B$. Regarded as a sequence to sequence operator, $B$ is an isomorphism between $H$ and $Y$.

Mapping Theorem. The operator $B$ bas domain $H$, is a 1-1 linear map of $H$ onto $Y$, is bounded, and has a bounded inverse. Its inverse is the restriction of A to $Y$.

The Uniqueness Theorem and Expansion Theorem are almost trivial corollaries of the Mapping Theorem. We shall derive them from the Mapping Theorem before giving a proof for it.

Proof of the Uniqueness Theorem. Let $S(z)=\sum_{k=0}^{\infty} b_{k} p_{k}(z)$ converge for all $z$, and set $y=\left\{S^{(j)}(0)\right\}_{0}^{\infty}$. The convergence is necessarily uniform on compact sets; therefore $y=B b$, so that $y \in Y$ and $S \in \mathcal{F}$. The sequence $b$ belongs to $H$, and the Mapping Theorem guarantees that $A y=A(B b)=b$. Therefore $L_{k}(S)=$ $(A y)_{k}=b_{k}, k=0,1, \cdots$ and the proof is complete.

Proof of the Expansion Theorem. We have already noted that convergence with respect to $\|\cdot\|$ is stronger than uniform convergence on compact sets; therefore we need only show that

$$
\lim _{n \rightarrow \infty}\left\|f-\sum_{k=0}^{n} L_{k}(f) p_{k}\right\|=0
$$

for every $f \in \mathcal{F}$. In view of our isometry, this is equivalent to 


$$
\lim _{n \rightarrow \infty}\left\|y-\sum_{k=0}^{n}(A y)_{k} B e_{k}\right\|_{Y}=0
$$

for every $y \in Y$.

Suppose $y \in Y$, and let $b=A y$. Then $b \in H$ and $B(A y)=y$ from the Mapping Theorem. Therefore

$$
\begin{aligned}
y-\sum_{k=0}^{n}(A y)_{k} B e_{k} & =B(A y)-\sum_{k=0}^{n}(A y)_{k} B e_{k} \\
& =B\left\{A y-\sum_{k=0}^{n}(A y)_{k} e_{k}\right\}=B\left\{b-\sum_{k=0}^{n} b_{k} e_{k}\right\} .
\end{aligned}
$$

Since the operator $B$ is bounded, we need only show that

$$
\lim _{n \rightarrow \infty}\left\|b-\sum_{k=0}^{n} b_{k} e_{k}\right\|_{H}=0
$$

Since

$$
\left\|b-\sum_{k=0}^{n} b_{k} e_{k}\right\|_{H}=\max _{1 \leq q \leq \lambda} \sup _{n<j<\infty}\left|\sum_{k=j}^{\infty}\left(\begin{array}{c}
k+m(q)-1 \\
m(q)-1
\end{array}\right) b_{k} \alpha_{q}^{k}\right|,
$$

(3.5) is obvious, and the proof is complete.

The proof of the Mapping Theorem, while long and fairly difficult, splits naturally into four parts. It is easy to verify that the Mapping Theorem is equivalent to the following four theorems, taken together.

Theorem 3.1. $B$ is a bounded linear map of $H$ into $Y$, and $H$ is the domain of $B$.

Theorem 3.2. $A(B b)=b$ for all $b \in H$.

Theorem 3.3. $B(A y)=y$ for all $y \in Y$.

Theorem 3.4. $A$ is a bounded linear map of $Y$ onto $H$.

In passing, we note that in terms of the power series coefficients of $\phi(z)=$ $\Sigma_{0}^{\infty} b_{j} z^{j}$, the matrix $B$ is given by

$$
B=\left[\begin{array}{cccc}
b_{0}, & b_{1}, & b_{2}, & \ldots \\
0, & b_{0}, & b_{1}, & \ldots \\
0, & 0, & b_{0}, & \ldots \\
\ldots & \ldots & \ldots & \ldots
\end{array}\right] .
$$


The same representation holds for $A$ in terms of the power series coefficients for $\theta(z)=\Sigma_{0}^{\infty} a_{j} z^{j}$. The matrices $A$ and $B$ are formal matrix inverses of each other; more generally, if

$$
\left\{\sum_{j=0}^{\infty} u_{j} z^{j}\right\}\left\{\sum_{j=0}^{\infty} v_{j} z^{j}\right\}=\sum_{j=0}^{\infty} w_{j} z^{j}
$$

is a formal power series identity, then

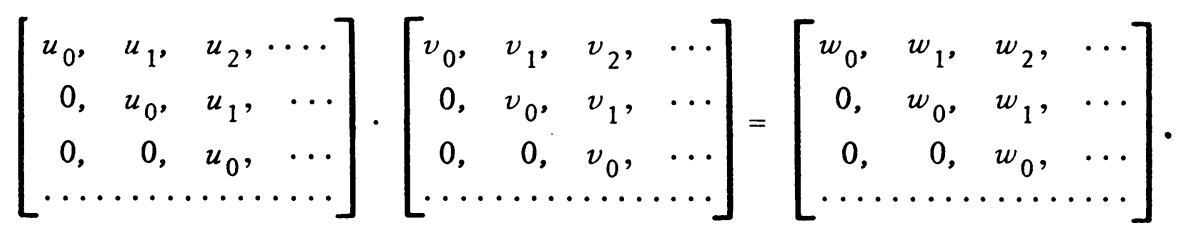

We shall need to consider a number of matrices of this form; for convenience we list them here. Let $g(z)=\Sigma_{0}^{\infty} u_{j} z^{j}$ and

$$
G=\left[\begin{array}{cccc}
u_{0}, & u_{1}, & u_{2}, & \ldots \\
0, & u_{0}, & u_{1}, & \ldots \\
0, & 0, & u_{0}, & \ldots \\
\ldots & \ldots & \ldots & \ldots
\end{array}\right]
$$

The following table lists the special matrices we use:

\begin{tabular}{|c|c||c|c|}
\multicolumn{1}{c}{$g(z)$} & \multicolumn{1}{c}{$G$} & \multicolumn{1}{c}{$g(z)$} & $G$ \\
\hline$\theta(z)$ & $A$ & $P_{m-1}(z)$ & $A^{\prime \prime}$ \\
$\phi(z)$ & $B$ & $1 / P_{m-1}(z)$ & $B^{\prime \prime}$ \\
$P_{m}(z)$ & $A^{\prime}$ & $P_{m-2}(z)$ & $A^{\prime \prime \prime}$ \\
$1 / P_{m}(z)$ & $B^{\prime}$ & $(1-\alpha z)^{-t}$ & $\nabla_{a}^{\prime}$ \\
\hline
\end{tabular}

Note that the conditions that $y \in Y$ can be written as

$$
\lim _{n \rightarrow \infty} \frac{y_{n}}{r^{n}}=0 \text { and } \quad \lim _{n \rightarrow \infty} \frac{n^{m-1}}{r^{n}}\left(A^{\prime \prime} y\right)_{n}=0 .
$$

Lemma 3.1. The domain of $B$ is $H$.

Proof. Suppose $b \in H$. Then $S(z)=\sum_{k=0}^{\infty} b_{k} p_{k}(z)$ is uniformly convergent on $|z| \leq 1$. For each nonnegative integer $j$, termwise differentiation $j$ times yields $S^{(j)}(z)=\Sigma_{k=j}^{\infty} b_{k} p_{k}^{(j)}(z)$. In particular, 


$$
S^{(i)}(0)=\sum_{k=j}^{\infty} b_{k} p_{k}^{(j)}(0)=\sum_{k=j}^{\infty} b_{k-j} b_{k}
$$

Therefore $b$ belongs to the domain of $B$.

Suppose now that $b$ belongs to the domain of $B$. Then $\sum_{k=j}^{\infty} b_{k} p_{k}^{(j)}(0)=$ $\sum_{k=j}^{\infty} b_{k-j} b_{k}$ converges for every nonnegative $j$. In particular, this is true if $0 \leq j$ $<m \lambda$; therefore $b \in H$ by the Convergence Theorem.

Lemma 3.2. If $j, s$ and $t$ are nonnegative integers, and $t \leq s$, then the total variation of

$$
\left\{\left(\begin{array}{c}
k+t \\
t
\end{array}\right)\left(\begin{array}{c}
k+j+s \\
s
\end{array}\right)^{-1}\right\}_{k=0}^{\infty}
$$

does not exceed $3\left(\begin{array}{c}s \\ t\end{array}\right)\left(\begin{array}{c}j+s \\ s-t\end{array}\right)^{-1}$.

Proof. Let $x_{k}$ denote the $k$ th term of (3.6). After some simplification, we obtain

$$
x_{k}-x_{k-1}=x_{k-1}\{(t j-k(s-t)) / k(k+j+s)\}, \quad k \geq 1 .
$$

Therefore $\left\{x_{k}-x_{k-1}\right\}_{1}^{\infty}$ changes sign at most once, and $\sum_{k=1}^{\infty}\left|x_{k}-x_{k-1}\right| \leq 2 K$, where $K=\sup _{0 \leq k<\infty} x_{k}$. Since $x_{0} \leq K$, the result follows if we establish that $K \leq\left(\begin{array}{c}s \\ t\end{array}\right)\left(\begin{array}{l}j+s \\ s-t\end{array}\right)-1$. Now

$$
\begin{aligned}
x_{k} & =\frac{s !}{t !}\left\{\left(\frac{k+1}{k+j+1}\right) \cdots\left(\frac{k+t}{k+j+t}\right)\right\} \frac{1}{(k+j+t+1) \cdots \cdots(k+j+s)} \\
& \leq \frac{s !}{t !(s-t) !}\left\{\frac{(s-t) !}{(k+j+t+1) \cdots(k+j+s)}\right\}=\left(\begin{array}{l}
s \\
t
\end{array}\right)\left(\begin{array}{c}
k+j+s \\
s-t
\end{array}\right)^{-1} \\
& \leq\left(\begin{array}{l}
s \\
t
\end{array}\right)\left(\begin{array}{c}
j+s \\
s-t
\end{array}\right)^{-1},
\end{aligned}
$$

which completes the proof.

Lemma 3.3. If $1 \leq q \leq \lambda, 1 \leq t \leq m(q)$, and $j$ is a nonnegative integer, then

$$
r^{-j}\left(\begin{array}{c}
j+m(q)-1 \\
m(q)-t
\end{array}\right)\left|\left(\nabla_{\alpha_{q}}^{t} b\right)_{j}\right| \leq 3\left(\begin{array}{c}
m-1 \\
t-1
\end{array}\right)^{2}\left\|b-\sum_{k=0}^{j-1} b_{k} e_{k}\right\|_{H}
$$

for all $b \in H$. In particular,

$$
r^{-i}\left|\left(\nabla_{a_{q}}^{t} b\right)_{j}\right| \leq 2^{2 m}\left\|b-\sum_{k=0}^{j-1} b_{k} e_{k}\right\|_{H} \leq 2^{2 m}\|b\|_{H} .
$$


Proof. We have

$$
\left(\nabla_{\alpha_{q}}^{t} b\right)_{j}=\sum_{k=j}^{\infty}\left(\begin{array}{c}
k-j+t-1 \\
t-1
\end{array}\right) b_{k} \alpha_{q}^{k-j}
$$

so that

$$
\alpha_{q}^{j}\left(\nabla_{q}^{t} b\right)_{j}=\sum_{k=j}^{\infty}\left(\begin{array}{c}
k+m(q)-1 \\
m(q)-1
\end{array}\right) b_{k} \alpha_{q}^{k}\left\{\left(\begin{array}{c}
k-j+t-1 \\
t-1
\end{array}\right)\left(\begin{array}{c}
k+m(q)-1 \\
m(q)-1
\end{array}\right)^{-1}\right\} .
$$

From Lemma 2.7 we obtain

$$
r^{-j}\left|\left(\nabla_{a_{q}}^{:} b\right)_{j}\right| \leq V_{j} \sup _{n \geq j}\left|\sum_{k=n}^{\infty}\left(\begin{array}{c}
k+m(q)-1 \\
m(q)-1
\end{array}\right) b_{k} \alpha_{q}^{k}\right|
$$

where $V_{j}$ denotes the total variation of the sequence

$$
\left\{\left(\begin{array}{c}
k-j+t-1 \\
t-1
\end{array}\right)\left(\begin{array}{c}
k+m(q)-1 \\
m(q)-1
\end{array}\right)^{-1}\right\}_{k=j}^{\infty}
$$

Now

$$
\begin{aligned}
\sup _{n \geq j}\left|\sum_{k=n}^{\infty}\left(\begin{array}{c}
k+m(q)-1 \\
m(q)-1
\end{array}\right) b_{k} \alpha_{q}^{k}\right| & \leq \max _{1 \leq q \leq \lambda} \sup _{n \geq j}\left|\sum_{k=n}^{\infty}\left(\begin{array}{c}
k+m(q)-1 \\
m(q)-1
\end{array}\right) b_{k} \alpha_{q}^{k}\right| \\
& =\left\|b-\sum_{k=0}^{j} b_{k} e_{k}\right\|_{H} .
\end{aligned}
$$

From Lemma 3.2 we see that $V_{j}$ does not exceed

$$
\begin{aligned}
3\left(\begin{array}{c}
m(q)-1 \\
t-1
\end{array}\right)\left(\begin{array}{c}
j+m(q)-t \\
m(q)-t
\end{array}\right)^{-1} & \leq 3\left(\begin{array}{c}
m(q)-1 \\
t-1
\end{array}\right)^{2}\left(\begin{array}{c}
j+m(q)-1 \\
m(q)-t
\end{array}\right)^{-1} \\
& \leq 3\left(\begin{array}{c}
m-1 \\
t-1
\end{array}\right)^{2}\left(\begin{array}{c}
j+m(q)-1 \\
m(q)-t
\end{array}\right)^{-1} .
\end{aligned}
$$

This proves the first half of Lemma 3.3. The second half follows easily.

Lemma 3.4. Let $\Sigma_{0}^{\infty} d_{k} z^{k}$ be analytic in $|z| \leq r$, and let

$$
M_{j k}= \begin{cases}d_{k-j}, & 0 \leq j \leq k<\infty \\ 0, & k<j .\end{cases}
$$

There is a positive constant $K$ such that, if $b \in H$ and $j$ is a nonnegative integer, then 


$$
r^{-j}\left(\begin{array}{c}
j+m-1 \\
m-1
\end{array}\right)\left|(M b)_{j}\right| \leq K\left\|b-\sum_{k=0}^{j-1} b_{k} e_{k}\right\|_{H} \leq K\|b\|_{H} .
$$

Proof. Choose $q$ so that $m(q)=m$. Then

$$
(M b)_{j}=\sum_{k=j}^{\infty} d_{k-j} b_{k}=\sum_{k=j}^{\infty}\left(\begin{array}{c}
k+m-1 \\
m-1
\end{array}\right) \alpha_{q}^{k} b_{k}\left\{\left\{\alpha_{q}^{-k} d_{k-j}\left(\begin{array}{c}
k+m-1 \\
m-1
\end{array}\right)^{-1}\right\} .\right.
$$

As before, we have $\left|(M b)_{j}\right| \leq V_{j}\left\|b-\Sigma_{k=0}^{j-1} b_{k} e_{k}\right\|_{H}$, where, in this instance, $V_{j}$ denotes the total variation of

$$
\left\{\dot{d}_{k-j} \alpha_{q}^{-k}\left(\begin{array}{c}
k+m-1 \\
m-1
\end{array}\right)^{-1}\right\}_{k=j}^{\infty}
$$

If $x$ and $y$ are sequences of bounded variation, the total variation of $\left\{x_{k} y_{k}\right\}_{0}^{\infty}$ does not exceed twice the product of the total variations of $x$ and $y$. Therefore the total variation of (3.7) does not exceed $4\left(\begin{array}{c}j+m-1 \\ m-1\end{array}\right)^{-1} V_{j}^{\prime}$, where $V_{j}^{\prime}$ denotes the total variation of $\left\{\alpha_{q}^{-k} d_{k-j}\right\}_{k=i}^{\infty}$. From the triangle in equality we obtain

$$
V_{j}^{\prime} \leq 2 \sum_{k=j}^{\infty}\left|\alpha_{q}^{-k} d_{k-j}\right|=2 r^{j} \sum_{k=0}^{\infty}\left|d_{k}\right| r^{k}
$$

If we take $K=8 \sum_{k=0}^{\infty}\left|d_{k}\right| r^{k}$, the result follows.

Lemma 3.5. $A^{\prime \prime}(B b)=\left(A^{\prime \prime} B\right) b$ for all $b \in H$.

Proof. For each nonnegative integer $n$ we have

$$
\left\{A^{\prime \prime}(B b)\right\}_{n} \cdot=\sum_{k=n}^{\infty} A_{n k}^{\prime \prime}(B b)_{k}=\sum_{k=n}^{n+\tau} A_{n k}^{\prime \prime}(B b)_{k},
$$

since $A_{n k}^{\prime \prime}=0$ for $k>n+\tau$ (recall that $\tau$ is the degree of $P_{m-1}$ ). Therefore

$$
\left\{A^{\prime \prime}(B b)\right\}_{n}=\sum_{k=n}^{n+\tau} A_{n k}^{\prime \prime} \sum_{j=k}^{\infty} B_{k j} b_{j}=\sum_{i=n}^{\infty} b_{j} \sum_{k=n}^{t(j, n)} A_{n k}^{\prime \prime} B_{k j}
$$

where $t(j, n)=\min \{n+\tau, j\}$. Since $A_{n k}^{\prime \prime}=0$ for $k>n+\tau$, we have

$$
\sum_{k=n}^{t(j, n)} A_{n k}^{\prime \prime} B_{k j}=\sum_{k=n}^{j} A_{n k}^{\prime \prime} B_{k j}=\left(A^{\prime \prime} B\right)_{n j} .
$$

Therefore $\left\{A^{\prime \prime}(B b)\right\}_{n}=\left\{\left(A^{\prime \prime} B\right) b\right\}_{n}$, which completes the proof. 
Lemma 3.6. There is a positive constant $K_{1}$ sucb that, if $j$ is a nonnegative integer,

$$
r^{-j}\left(\begin{array}{c}
j+m-1 \\
m-1
\end{array}\right)\left|\left\{\left(A^{\prime \prime} B\right) b\right\}_{j}\right| \leq K_{1}\left\|b-\sum_{k=0}^{j-1} b_{k} e_{k}\right\|_{H} \leq K_{1}\|b\|_{H} .
$$

Proof. Set $\phi_{3}(z)=P_{m-1}(z) \phi(z)=\sum_{j=0}^{\infty} b_{j}^{\prime \prime} z^{j}$. Then

$$
\left(A^{\prime \prime} B\right)_{k j}= \begin{cases}b_{j-k}^{\prime \prime}, & 0 \leq k \leq j<\infty \\ ?, & k>j .\end{cases}
$$

The function $\phi_{3}$ has only simple poles on the circle $|z|=r$. Therefore we can write

$$
\phi_{3}(z)=\sum_{m(q)=m} \frac{c^{\prime \prime}(q)}{1-\alpha_{q} z}+\phi_{4}(z)
$$

where the $c^{\prime \prime}(q)$ are complex numbers and $\phi_{4}$ is analytic in $|z| \leq r$. The corresponding matrix equation is $A^{\prime \prime} B=\Sigma_{m(q)=m} c^{\prime \prime}(q) \nabla_{a_{q}}+M "$, where

$$
M_{k j}^{\prime \prime}=\left\{\begin{array}{l}
\phi_{4}^{(j-k)}(0) /(j-k) !, \quad 0 \leq j \leq k<\infty, \\
0, \quad k>j .
\end{array}\right.
$$

The result now follows from Lemma 3.3 (with $t=1$ ) and Lemma 3.4.

Proof of Theorem 3.1. The matrix equation corresponding to the decomposition

$$
\phi(z)=\sum_{q=1}^{\lambda} \sum_{t=1}^{m(q)} \frac{c_{t}(q)}{\left(1-\alpha_{q} z\right)^{m(q)+1-t}}+\phi_{2}(z)
$$

is given by

$$
B=\sum_{q=1}^{\lambda} \sum_{t=1}^{m(q)} c_{t}(q) \nabla_{q}^{m(q)+1-t}+M
$$

where

$$
M_{k j}=\left\{\begin{array}{l}
\phi_{2}^{(j-k)}(0) /(j-k) !, \quad 0 \leq k \leq j<\infty, \\
0, \quad k>j .
\end{array}\right.
$$

If $b \in H$, then $b$ belongs to the domain of every matrix in (3.8), and we have

$$
B b=\sum_{q=1}^{\lambda} \sum_{t=1}^{m(q)} c_{t}(q) \nabla_{a}^{m(q)+1-t} b+M b .
$$


It follows from Lemmas 3.3 and 3.4 that $\lim _{j \rightarrow \infty} r^{-j}(B b)_{j}=0$, and from Lemma 3.6

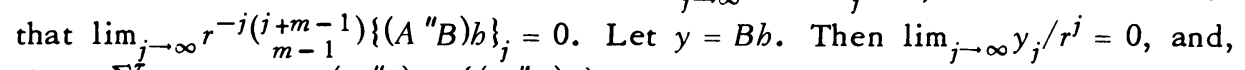
since $\sum_{k=0}^{\tau} C_{k} y_{k+j}=\left(A^{\prime \prime} y\right)_{j}=\left\{\left(A^{\prime \prime} B\right) b\right\}_{j}$, we have

$$
\lim _{j \rightarrow \infty} r^{-j}\left(\begin{array}{c}
j+m-1 \\
m-1
\end{array}\right) \sum_{k=0}^{\tau} C_{k} y_{k+j}=0 .
$$

Therefore $y \in Y$. The proof that $B$ is bounded follows similarly from Lemmas 3.3, 3.4, and 3.6, and this completes the proof of Theorem 3.1.

Lemma 3.7. Let $g_{1}$ and $g_{2}$ be analytic in $|z| \leq r$ and, for $i=1,2$, set

$$
\left(G^{(i)}\right)_{j k}=\left\{\begin{array}{l}
g_{i}^{(k-j)}(0) /(k-j) !, \quad 0 \leq j \leq k<\infty, \\
0, \quad j>k .
\end{array}\right.
$$

If the sequence $x$ satisfies $\lim _{j \rightarrow \infty} x_{j} / r^{j}=0$, then $G^{(1)}\left(G^{(2)} x\right)=\left(G^{(1)} G^{(2)}\right) x$.

Proof. For each $n \geq 0$ we have

$$
\left\{G^{(1)}\left(G^{(2)} x\right)\right\}_{n}=\sum_{j=n}^{\infty} G_{n j}^{(1)}\left(G^{(2)} x\right)_{j}=\sum_{j=n}^{\infty} G_{n j}^{(1)} \sum_{k=j}^{\infty} G_{j k}^{(2)} x_{k} .
$$

Now

$$
\sum_{i=i}^{\infty}\left|G_{j k}^{(2)} x_{k}\right|=r^{j} \sum_{k=i}^{\infty} \frac{g_{2}^{(k-i)(0)}}{(k-j) !} \frac{\left|x_{k}\right|}{r^{k}} r^{k-j} \leq K r^{j},
$$

where $K=\left\{\sup _{0 \leq k \leq \infty}\left|x_{k}\right| / r^{k}\right\} \sum_{k=0}^{\infty}\left|g_{2}^{(k)}(0)\right| r^{k} / k !$. Therefore

$$
\sum_{j=n}^{\infty}\left|G_{n j}^{(1)}\right| \sum_{k=j}^{\infty}\left|G_{j k}^{(2)} x_{k}\right| \leq K \sum_{j=n}^{\infty}\left|G_{n j}^{(1)}\right| r^{j}=K r^{n} \sum_{j=n}^{\infty} \frac{\left|g_{1}^{(j-n)}(0)\right|}{(j-n) !} r^{j-n}<\infty .
$$

This allows us to interchange the order of summation in (3.9) and obtain

$$
\left\{G^{(1)}\left(G^{(2)} x\right\}_{n}=\sum_{k=n}^{\infty} x_{k} \sum_{j=n}^{k} G_{n j}^{(1)} G_{j k}^{(2)}=\left\{\left(G^{(1)} G^{(2)}\right) x\right\}_{n}\right.
$$

this completes the proof.

Proof of Theorem 3.2. Suppose $b \in H$. Then $A^{\prime}(B b)=\left(A^{\prime} B\right) b$ by an argument identical to the proof of Lemma 3.5. In Lemma 3.7, take $G^{(1)}=A B^{\prime}$, $G^{(2)}=A^{\prime}$, and $x=B b$. We obtain $\left(A B^{\prime}\right)\left\{A^{\prime}(B b)\right\}=\left\{\left(A B^{\prime}\right) A^{\prime}\right\}(B b)=A(B b)$. In Lemma 3.7, take $G^{(1)}=A B^{\prime}, G^{(2)}=A^{\prime} B$, and $x=b$. We obtain $\left(A B^{\prime}\right)\left\{\left(A^{\prime} B\right) b\right\}=$ $\left\{\left(A B^{\prime}\right)\left(A^{\prime} B\right)\right\} b=b$, and the proof is complete. 
Lemma 3.8. Let $R_{0}$ denote the space of all sequences $y$ such that $\lim _{j \rightarrow \infty} y_{j} / r^{j}=0 ;\|y\|_{R_{0}}=\sup _{0 \leq j<\infty}\left|y_{j}\right| / r^{j}$. The restriction of $A$ to $R_{0}$ is a 1-1 bounded linear map of $R_{0}$ into $R_{0}$.

Proof. That $A$ is a bounded linear map of $R_{0}$ into $R_{0}$ follows easily from the fact that $A$ is upper triangular, together with

$$
\sum_{k=j}^{\infty}\left|A_{j k}\right| r^{k}=\sum_{k=j}^{\infty}\left|a_{k-j}\right| r^{k}=r^{j} \sum_{k=0}^{\infty}\left|a_{k}\right| r^{k} .
$$

For all $y \in R_{0}$, we have $A y=A^{\prime}\left(\left(B^{\circ} A\right) y\right)$ by Lemma 3.7. The previous argument implies that both $A^{\prime}$ and $B^{\prime} A$ are bounded linear maps of $R_{0}$ into $R_{0}$. Again from Lemma 3.7, we have $\left(A B^{\prime}\right)\left\{\left(B^{\prime} A\right)_{y}\right\}=y, y \in R_{0}$. Therefore $B^{\prime} A$ restricted to $R_{0}$ is $1-1$. To complete the proof, we need only show that the same is true of $A^{\prime}$.

If $P$ is of degree 1 , then $P(z)=1-\alpha_{1} z$ and $A^{\prime}$ is the matrix

$$
\Delta_{a_{1}}=\left[\begin{array}{rrrrr}
1, & -a_{1}, & 0, & 0, & \ldots \\
0, & 1, & -a_{1}, & 0, & \ldots \\
0, & 0, & 1, & -a_{1}, & \ldots \\
\ldots \ldots & \ldots \ldots
\end{array}\right]
$$

The solutions of $\Delta_{\alpha_{1}} y=0$ are the constant multiples of the sequence $\left\{\alpha_{1}^{-j}\right\}_{0}^{\infty}$. Therefore $\Delta_{a_{1}}$ is a 1-1 map of $R_{0}$ into $R_{0}$. The set of linear 1-1 maps of $R_{0}$ into $R_{0}$ is closed under composition. The extension of Lemma 3.7 to an arbitrary number of factors is immediate, and since $A^{\prime}$ has the matrix factorization $A^{\prime}=$ $\Pi_{q=1}^{\lambda}\left(\Delta_{\alpha_{q}}\right)^{m(q)}$, the proof is complete.

The point of Lemma 3.8 is that $y \in R_{0}$ and $A y \in H$ imply that $B(A y)=y$. To see this, set $x=B(A y)$. Then $x \in R_{0}$ by Theorem 3.1, and from Theorem 3.2 we obtain $A x=A(B(A y))=A y$; therefore $x=y$. We note for future use that $A^{\prime \prime}$ restricted to $R_{0}$ is $1-1$; the proof is the same as that for $A^{\prime}$.

Lemma 3.9. If $n$ and $s$ are integers, $n \geq 0, s \geq 2$, then

$$
\sum_{k=n}^{\infty}\left(\begin{array}{c}
k+s \\
s
\end{array}\right)^{-1}=\frac{s}{s-1}\left(\begin{array}{c}
n+s-1 \\
s-1
\end{array}\right)^{-1} .
$$

Proof. This follows easily from the binomial coefficient identity

$$
\left(\begin{array}{c}
k+s \\
s
\end{array}\right)^{-1}=\frac{s}{s-1}\left\{\left(\begin{array}{c}
k-1+s \\
s-1
\end{array}\right)^{-1}-\left(\begin{array}{c}
k+s \\
s-1
\end{array}\right)^{-1}\right\} .
$$


Lemma 3.10. Suppose that $m>2$ and that $|\alpha|=r$. If the sequence $x$ satisfies $\lim _{j \rightarrow \infty} r^{-j} j^{m-1} x_{j}=0$, then

$$
\lim _{j \rightarrow \infty} r^{-j} j^{m-2}\left(\nabla_{\alpha} x\right)_{j}=0 .
$$

Proof. For each $j,\left(\nabla_{\alpha^{x}}\right)_{j}=\sum_{k=j}^{\infty} \alpha^{k-j} x_{k}$. Therefore

$$
\begin{aligned}
r^{-j}\left|\left(\nabla_{a} x\right)_{j}\right| & =\left|\sum_{k=j}^{\infty}\left(\begin{array}{c}
k+m-1 \\
m-1
\end{array}\right) \alpha^{k} x_{k}\left(\begin{array}{c}
k+m-1 \\
m-1
\end{array}\right)^{-1}\right| \\
& \leq \sup _{k \geq j}\left\{\left(\begin{array}{c}
k+m-1 \\
m-1
\end{array}\right) r^{-k}\left|x_{k}\right|\right\} \sum_{k=j}^{\infty}\left(\begin{array}{c}
k+m-1 \\
m-1
\end{array}\right)^{-1} .
\end{aligned}
$$

Using Lemma 3.9, we obtain

$$
\left(\begin{array}{c}
j+m-2 \\
m-2
\end{array}\right) r^{-j}\left|\left(\nabla_{a} x\right)\right| \leq \frac{m-1}{m-2} \sup _{j \geq j}\left\{\left(\begin{array}{c}
k+m-1 \\
m-1
\end{array}\right) r^{-k}\left|x_{k}\right|\right\},
$$

which implies (3.10).

Lemma 3.11. Suppose that $m>2$. If the sequence $y$ is such that $y \in R_{0}$ and $\lim _{j \rightarrow \infty} r^{-j} j^{m-1}\left(A^{\prime \prime} y\right)_{j}=0$, then $\lim _{j \rightarrow \infty} r^{-j} j^{m-2}\left(A^{\prime \prime \prime} y\right)_{j}=0$.

Proof. Let $x=A^{\prime \prime} y$ and $G=A^{\prime \prime \prime} B^{\prime \prime}$. The matrix $G$ corresponds to the analytic function

$$
g(z)=\frac{P_{m-2}(z)}{P_{m-1}(z)}=\prod_{m(q)>m-1}\left(1-\alpha_{q} z\right)^{-1},
$$

which has only simple poles on $|z|=r$. Since $m>2$, the hypothesis on $A$ " $y$ insures that $x$ belongs to the domain of $G$; set $w=G x=G\left(A^{\prime \prime} y\right)$. Then

$$
A^{\prime \prime} w=A^{\prime \prime}(G x)=\left(A^{\prime \prime} G\right) x=A^{\prime \prime \prime} x=A^{\prime \prime \prime}\left(A^{\prime \prime} y\right)=\left(A^{\prime \prime \prime} A^{\prime \prime}\right) y=A^{\prime \prime}\left(A^{\prime \prime \prime} y\right) \text {. }
$$

It follows from the argument used to prove Lemma 3.3 that the range of $G$ is contained in $R_{0}$; therefore $w \in R_{0}, A^{\prime \prime} y \in R_{0}$, and the remark following Lemma 3.8 insures that $A^{\prime \prime}$ is $1-1$ on $R_{0}$. Therefore $G x=A^{\prime \prime \prime} y$, and it only remains to show that

$$
\lim _{j \rightarrow \infty} r^{-j} j^{m-2}(G x)_{j}=0 .
$$

The partial fraction decomposition of $g$ corresponds to a matrix identity which expresses $G$ as a linear combination of the matrices $\nabla_{a_{q}}, m(q) \geq m-1$. We then obtain (3.11) from Lemma 3.10.

It is crucial for the proof of Theorem 3.3 to know that the corresponding 
result is true in a slightly different space. For $m>1$, denote $Y$ by $Y(P)$ (the space is completely determined by the polynomial $P$ ), and let $Y\left(P_{m-1}\right)$ be the space obtained by replacing $P$ by $P_{m-1}$; this is equivalent to replacing $\phi$ by $1 / P_{m-1}$. It is trivial to show that $y$ belongs to $Y\left(P_{m-1}\right)$ if and only if $\lim _{j \rightarrow \infty} r^{-j} y_{j}=0$ and $\lim _{j \rightarrow \infty} r^{-j} j^{m-2}\left(A^{\prime \prime \prime} y\right)_{j}=0$. The purpose of Lemmas 3.9, 3.10 , and 3.11 was to establish that every point of $Y=Y(P)$ belongs to $Y\left(P_{m-1}\right)$, if $m>2$. For $m=2$, the result is obvious.

Lemma 3.12. If $x$ is in the domain of $B^{\prime \prime}$ and $\lim _{n \rightarrow \infty} r^{-n} n^{m-1} x_{n}=0$, then $B\{(A B ") x\}=B " x$.

Proof. Let $s$ be a nonnegative integer and set $G=A B "$. For $n \geq s$, we have

$$
\sum_{k=s}^{n}(G x)_{k} B_{s k}=\sum_{k=s}^{n}\left\{\sum_{j=k}^{\infty} G_{k j} x_{j}\right\} B_{s k}=\sum_{j=s}^{\infty} x_{j} \sum_{k=s}^{\min \{n, j\}} G_{k j} B_{s k} .
$$

If we split the last series at $j=n$, we obtain

$$
\sum_{j=s}^{n} x_{j} \sum_{k=s}^{j} G_{k j} B_{s k}+\sum_{j=n+1}^{\infty} x_{j} \sum_{k=s}^{n} G_{k j} B_{s k} .
$$

But $\Sigma_{k=s}^{j} G_{k j} B_{s k}=(B G)_{s j}=B_{s j}^{\prime \prime}$. Therefore

$$
\sum_{k=s}^{n}(G x)_{k} B_{s k}-\sum_{j=s}^{n} B_{s j}^{\prime \prime} x_{j}=\sum_{j=n+1}^{\infty} x_{j} \sum_{k=s}^{n} G_{k j} B_{s k} .
$$

By hypothesis, $\left(B^{\prime \prime} x_{s}=\sum_{j=s}^{\infty} B_{s j}^{\prime \prime} x_{j}\right.$ converges. We shall prove that $\{B(G x)\}_{s}=$ $\sum_{k=s}^{\infty}(G x)_{k} B_{s k}$ converges to $\left(B^{\prime \prime} x_{s}\right.$ by showing that

$$
\lim _{n \rightarrow \infty} \sum_{j=n+1}^{\infty} x_{j} \sum_{k=s}^{n} G_{k j} B_{s k}=0 .
$$

Let $g(z)=\theta(z) / P_{m-1}(z)=\sum_{k=0}^{\infty} u_{k} z^{k}$. Note that $g$ is analytic in $|z| \leq r$ and that $g$ corresponds to the matrix $G=A B^{\prime \prime}$. Since $B_{s k}=\phi^{(k-s)}(0) /(k-s)$ !, $k \geq s$, and the only singularities of $\phi$ on $|z|=r$ are poles of order $m$ or less, there is a constant $K$ such that $\left|B_{s k}\right| \leq K r^{s-k}(k+1-s)^{m-1}$ for $0 \leq s \leq k<\infty$. For $s \leq k \leq n$ we have $\left|B_{s k}\right| \leq K(n+1)^{m-1} r^{s-k}$. Therefore

$$
\begin{aligned}
& \left|\sum_{i=n+1}^{\infty} x_{j} \sum_{k=s}^{n} u_{j-k} B_{s k}\right| \leq \sum_{j=n+1}^{\infty}\left|x_{j}\right| \sum_{k=s}^{n}\left|u_{j-k}\right| K(n+1)^{m-1} r^{s-k} \\
& \quad \leq K r^{s} \sum_{j=n+i}^{\infty} r^{-j}\left|x_{j}\right| j^{m-1} \sum_{k=s}^{n}\left|u_{j-k}\right| r^{j-k} \leq S_{n} K r^{s} \sum_{j=n+1}^{\infty} \sum_{k=s}^{\infty}\left|u_{j-k}\right| r^{j-k},
\end{aligned}
$$


where $S_{n}=\sup _{j>n} r^{-j} j^{m-1}\left|x_{j}\right| \cdot$ Now

$$
\sum_{j=n+1}^{\infty} \sum_{k=s}^{n}\left|u_{j-k}\right| r^{j-k}<\sum_{j=n+1}^{\infty} \sum_{k=-\infty}^{n}\left|u_{j-k}\right| r^{j-k}=\sum_{t=1}^{\infty} t\left|u_{t}\right| r^{t} .
$$

Since $S_{n} \rightarrow 0$ as $n \rightarrow \infty$, the proof is complete.

Proof of Theorem 3.3. Suppose $y \in Y$ and that $m=1$. In this case $B$ " is ths identity matrix, and we obtain $B(A y)=y$ from Lemma 3.12 by taking $x=y$.

We now have a basis for an argument by mathematical induction on $m$. Suppose that Theorem 3.3 is true for the space $Y\left(P_{m-1}\right)$, i.e., that

$$
B^{\prime \prime}\left(A^{\prime \prime} y\right)=y
$$

holds for all $y \in Y\left(P_{m-1}\right)$.

Let $y$ be a point of $Y=Y(P)$. From the discussion following Lemma 3.11 it follows that $y \in Y\left(P_{m-1}\right)$; therefore (3.12) holds. Let $x=A^{\prime \prime} y$. Then $y=$ $B^{\prime \prime}\left(A^{\prime \prime} y\right)=B^{\prime \prime} x$, so that $x$ belongs to the domain of $B "$. Since $y \in Y$, we have

$$
0=\lim _{n \rightarrow \infty} r^{-n} n^{m-1}\left(A^{\prime \prime} y\right)_{n}=\lim _{n \rightarrow \infty} r^{-n} n^{m-1} x_{n},
$$

and we may apply Lemma 3.12. This yields $B\left\{\left(A B^{\prime \prime}\right) x\right\}=B^{\prime \prime} x=y$. On the other hand, $(A B$ " $) x=\left(A B^{\prime \prime}\right)\left(A^{\prime \prime} y\right)=\left\{\left(A B^{\prime \prime}\right) A^{\prime \prime}\right\} y=A y$ by Lemma 3.7. Therefore $B(A y)=y$ and the proof is complete.

Proof of Theorem 3.4. Theorems 3.1 and 3.2 imply that $H$ is a subset of the image of $Y$ under $A$. Theorems 3.1 and 3.3 imply that the image of $Y$ under $A$ is a subset of $H$. Therefore $A$ is a linear map of $Y$ onto $H$. To show that $A$ is bounded, we use the principle of uniform boundedness. For each nonnegative integer $s$, let

$$
A_{k j}^{(s)}= \begin{cases}A_{k j} & \text { if } 0 \leq k \leq s \\ 0 & \text { if } k>s\end{cases}
$$

Lemma 3.13. $A^{(s)}$ is a bounded linear map of $Y$ into $H$.

Lemma 3.14. If $y \in Y$, then $\lim _{s \rightarrow \infty}\left\|A y-A^{(s)} y\right\|_{H}=0$.

Lemma 3.15. $Y$ is complete.

The proofs of Lemmas 3.13, 3.14, and 3.15 are straightforward and involve nothing difficult; we omit them. These lemmas insure that the hypotheses of the uniform boundedness principle are satisfied; therefore $A$ is bounded and the proof is complete.

4. Proof of the Structure Theorem. The space $\mathcal{F}$ is clearly isomorphic to $Y$. The proof that $Y$ is isomorphic to $c_{0}$ requires a double application of the Mapping 
Theorem and a slight modification of the norm on $H$. For notational simplicity, we write "Max" to indicate a maximum taken over those integers $q$ such that $1 \leq q \leq \lambda$ and $m(q) \geq m-1$.

Lemma 4.1. For $b \in H$, let

$$
N(b ; H)=\operatorname{Max} \sup _{0 \leq n<\infty}\left|\sum_{k=n}^{\infty}\left(\begin{array}{c}
k+m(q)-1 \\
m(q)-1
\end{array}\right) b_{k} \alpha_{q}^{k}\right| .
$$

Then $N(; H)$ is a norm on $H$ which is equivalent to $\|\cdot\|_{H}$.

Proof. It is obvious from the definition of $N(; H)$ that $N(; H)$ is a norm and that

$$
\|b\|_{H} \geq N(b ; H)
$$

for all $b \in H$. If $m=1$ or $m=2$, equality holds in (4.1). Suppose that $m \geq 3$ and that the points $\left\{a_{q}\right\}_{1}^{\lambda}$ are ordered so that $m(1)=m$. If $m(q) \leq m-2$, we have

$$
\sum_{k=n}^{\infty}\left(\begin{array}{c}
k+m(q)-1 \\
m(q)-1
\end{array}\right) b_{k} \alpha_{q}^{k}=\sum_{k=n}^{\infty}\left(\begin{array}{c}
k+m-1 \\
m-1
\end{array}\right) b_{k} \alpha_{1}^{k} x_{k}
$$

where

$$
x_{k}=\left(\alpha_{q} / \alpha_{1}\right)^{k}\left(\begin{array}{c}
k+m(q)-1 \\
m(q)-1
\end{array}\right)\left(\begin{array}{c}
k+m-1 \\
m-1
\end{array}\right)^{-1}
$$

Therefore

$$
\left|\sum_{k=n}^{\infty}\left(\begin{array}{c}
k+m(q)-1 \\
m(q)-1
\end{array}\right) b_{k} a_{q}^{k}\right| \leq V(x) \sup _{j \geq n}\left|\begin{array}{c}
\infty \\
k=n
\end{array}\left(\begin{array}{c}
k+m-1 \\
m-1
\end{array}\right) b_{k} a_{1}^{k}\right| \leq V(x) N(b ; H)
$$

where $V(x)$ denotes the total variation of $\left\{x_{k}\right\}_{0}^{\infty}$.

Now

$$
\left|x_{k}\right| \leq\left(\begin{array}{c}
k+m-3 \\
m-3
\end{array}\right)\left(\begin{array}{c}
k+m-1 \\
m-1
\end{array}\right)^{-1}=\frac{(m-1)(m-2)}{(k+m-1)(k+m-2)} \leq \frac{(m-1)(m-2)}{(k+2)(k+1)},
$$

since $m \geq 3$. Therefore $V(x) \leq \sum_{k=0}^{\infty} 2\left|x_{k}\right| \leq 2(m-1)(m-2)$. Consequently,

$$
\max _{m(q) \leq m-2} \sup _{0 \leq n<\infty}\left|\sum_{k=n}^{\infty}\left(\begin{array}{c}
k+m(q)-1 \\
m(q)-1
\end{array}\right) b_{k} a_{q}^{k}\right| \leq 2(m-1)(m-2) N(b ; H) .
$$

Therefore $\|b\|_{H} \leq 2(m-1)(m-2) N(b ; H)$, and the proof is complete. We note for future use that convergence of 


$$
\sum_{k=n}^{\infty}\left(\begin{array}{c}
k+m(q)-1 \\
m(q)-1
\end{array}\right) b_{k} \alpha_{q}^{k}
$$

for those integers $q$ such that $m(q) \geq m-1$ is equivalent to the convergence of 4.2 for $1 \leq q \leq \lambda$.

Lemma 4.2. The space $Y$ remains unchanged if $\phi(z)$ is replaced by

$$
\phi(z)\left\{\prod_{m(q)=m-1}\left(1-\alpha_{q} z\right)^{-1}\right\} \text {. }
$$

Proof. The space. $Y$ is completely determined by the polynomial

$$
P_{m-1}(z)=\prod_{q=1}^{\lambda}\left(1-c_{q} z\right)^{\min \{m(q), m-1\}} .
$$

Replacing $\phi(z)$ by (4.3) has the effect of increasing by 1 the multiplicity of every zero of $P(z)$ whose multiplicity is $m-1$. In view of (4.4), this has no effect whatever on $P_{m-1}(z)$, and the proof is complete.

It is crucial to our proof of the Structure Theorem that the space $Y$ cannot distinguish between the zeros of $P(z)$ of multiplicity $m$ and those of multiplicity $m-1$. Let $H^{\prime}$ denote the space of all sequences $b$ such that $\sum_{k=0}^{\infty}\left(\begin{array}{c}k+m-1 \\ m-1\end{array}\right) b_{k} \alpha_{q}^{k}$ converges if $m(q) \geq m-1$. For $b \in H^{\prime}$, set

$$
\|b\|_{H^{\prime}}=\operatorname{Max} \sup _{0 \leq n<\infty}\left|\sum_{k=n}^{\infty}\left(\begin{array}{c}
k+m-1 \\
m-1
\end{array}\right) b_{k} \alpha_{q}^{k}\right| .
$$

The remark following Lemma 4.1 insures that $b \in H^{\prime}$ implies the convergence of

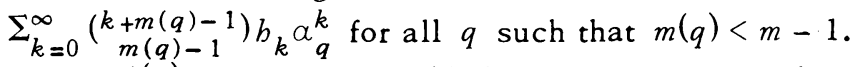

If $\phi(z)$ is replaced by (4.3), the space $Y$ remains unchanged, and $H$ is replaced by a corresponding space $H_{0}$. The sequences which belong to $H_{0}$ are the same as the sequences which belong to $H^{\prime}$ (the space $H_{0}$ differs from $H$ only in that $m(q)$ has been increased by 1 for those values of $q$ for which $m(q)=m-1)$. If we replace the norm on $H_{0}$ by the equivalent norm $N\left(; H_{0}\right)$, the space obtained is precisely $H^{\prime}$. Therefore $H^{\prime}$ is isomorphic to $H_{0}$, and from the Mapping Theorem we have that $H_{0}$ is isomorphic to $Y$.

Lemma 4.3. Let $H^{\prime \prime}$ denote the space of all sequences $b$ sucb that $\sum_{k=0}^{\infty} b_{k} \alpha_{q}^{k}$ converges if $m(q) \geq m-1$. For $b \in H^{\prime \prime}$, set

$$
\|b\|_{H^{\prime \prime}}=\operatorname{Max} \sup _{0 \leq n<\infty}\left|\sum_{k=n}^{\infty} b_{k} \alpha_{q}^{k}\right| .
$$

Then $H^{\prime}$ is isomorpbic to $H^{\prime \prime}$. 
Proof. Let $T$ be defined by $(T h)_{k}=\left(\begin{array}{c}k+m-1 \\ m-1\end{array}\right) b_{k}, b \in H^{\prime}, k=0,1,2, \ldots$, and note that $T$ is a linear isometry between $H^{\prime}$ and $H^{\prime \prime}$.

Lemma 4.4. $H^{\prime \prime}$ is isomorphic to $c_{0}$.

Proof. If $\phi(z)$ is replaced by $\left\{\Pi_{m(q) \geq m-1}\left(1-\alpha_{q} z\right)\right\}^{-1}$, it is easy to verify that $H$ is replaced by $H^{\prime \prime}, m$ is replaced by 1 and $Y$ is replaced by the space $Y^{\prime \prime}$ of all sequences $y$ such that $\lim _{n \rightarrow \infty} r^{-n} y_{n}=0 ;\|y\|_{Y} \prime=2 \sup _{0 \leq n<\infty} r^{-n}\left|y_{n}\right|$. The Mapping Theorem guarantees that $H^{\prime \prime}$ is isomorphic to $Y^{\prime \prime}$, and the isomorphism between $Y^{\prime \prime}$ and $c_{0}$ is obvious. Therefore $Y$ is isomorphic to $c_{0}$ and the proof of the Structure Theorem is complete.

\section{REFERENCES}

1. R. P. Boas, Jr., and R. C. Buck, Polynomial expansions of analytic functions, 2nd printing, corrected, Ergebnisse der Mathematik und ihrer Grenzgebiete, N. F., Band 19, Academic Press, New York; Springer-Verlag, Berlin, 1964. MR 29 \#218.

2. J. D. Buckholtz, Series expansions of analytic functions, J. Math. Anal. Appl. 41 (1973), 673-684.

3. W. T. Martin, On expansions in terms of a certain general class of functions, Amer. J. Math. 58 (1936), 407-420.

4. G. A. Read, Expansions in series of polynomials, J. London Math. Soc. 43 (1968), 655-657. MR $37 \# 4273$.

DEPARTMENT OF MATHEMATICS, UNIVERSITY OF KENTUCKY, LEXINGTON, KENTUCKY 40506 